\title{
Index of Signs and Symptoms
}

A Abdominal pain 106

Acanthosis nigricans 32, 98, 100

Acne 11, 32, 53

Adipomastia 21

Adrenal hyperandrogenism,

primary functional 32,34

Adrenal hyperplasia, congenita

after newborn period 52

in newborn period 50

nonclassical 18,34

Adrenal insufficiency 58, 62

Adrenal rests 34

Adrenal tumor 16

Adrenarche 18, 52

Adrenocorticotropic hormone, insensitivity 8

African-American 100

Albright osteodystrophy 11

Albumin 68

Aldosteronism 46, 66

Alopecia 32

Alström syndrome 10, 54

Amenorrhea

athletic, hypothalamic 30

primary, secondary 28,44

Androgen(s)

excess, signs 53

exogenous 16

insensitivity, incomplete 36,38

insensitivity syndrome 8

resistance 26

suppression 34

normal 34

subnormal 34

Anorexia 24, 58, 60

nervosa 25, 29

Anosmia 22, 24, 31

Anovulation 29, 32

Anovulatory disorders 30

Anthropometry 2

Aortic stenosis, supravalvular 69

Apocrine sweat odor 53

Appetite, uncontrollable 11

Aromatase

deficiency 9,40

inhibitors 16

Aromatization excess, familial 20

Athlete 24

Athletic amenorrhea 26, 30
Athyreosis 76

Autonomous nervous system, activation 95

Autosomal-dominant inheritance 104

B Bardet-Biedel syndrome 54

artter syndrome 66, 74

Beckwith-Wiedemann syndrome 8, 10, 94

Bicarbonaturia 62

Blue diaper syndrome 69

Body

fat, redistribution 48

mass index 10

odors, changed 19

Bone age $6,14,18,52$

advanced 16

Brain

damage 39

rradiation 12

Breast(s)

bloody discharge 21

development

delayed or absent 24

precocious 14

hard 21

irregular consistency 21

Burns 60

C Calcium levels

$$
\text { serum } 72
$$

urine 72

Calcium receptor defects 70

Caloric intake 10

Cardiac arrest 64

Cardiac insufficiency, mild 56

Carpenter syndrome 10

Central nervous system lesion 6

Chemotherapy 74

Chorionic gonadotropin-secreting

syndrome, human 14

tumors 16

Clitoral hypertrophy 19

Clitoromegaly 52

isolated, vaginal fusion 26

Coarctation 46

Cohen syndrome 10

Coma 106

Confusion 106

Conscious level, reduced 106
Conn syndrome 56,66

Convulsions 63

Corneal drying 81

Coronary heart diseases 11

Cortical suppression normal 34

Cortisol resistance and metabolic defects 34

Craniopharyngioma 10

Cryptorchidism 22, 38, 42

Cushing

disease 48

syndrome $10,22,32,34,48,56$

D Dehydration 50, 58, 106

fever 58

hypertonic 60

Diabetes

drug-induced 98

insipidus 58, 60

central 54

dipsogenic, partial, pituitary 54

maternal 94

maturity-onset of the young 96, 98, 100, 104 mellitus 54, 100, 104

immune-mediated 96, 98, 100, 104

non-immune-mediated $96,98,100$

Type 1 100, 102

Type 2 98, 100

Diabetic ketoacidosis 106

Diabetic mother 10

Diarrhea 58

chronic 74

profuse 66

Diencephalic syndrome 2

Diuresis, insufficient 56

Diuretic (ab) use 63

Diuretic excess 62

Dyshormonogenesis of thyroid $76,78,84,85$

E Ear lobe fissures 94

Eating disorder 30

Edema, female newborn 44

Electrolytes, urea 106

Elfin faces 69

Enterostomy losses 66

Estradiol 30

Estrogen

deficiency, insensitivity 8

receptor defects 9 
Ethnic background 100

high risk for diabetes 100

Ethnic groups 19

Eunuchoid habitus 9,22

Euthyroid 82

hyperthyroxinemia 92

Exophthalmos 80

F Failure to thrive 2

Fanconi syndrome(s) 66, 70, 72

distribution 10

subcutaneous 69

Feminizing disorders 21

Fever 80, 84

Fibrous dysplasia 72

First-degree relative

type 1 diabetes mellitus 98

type 2 diabetes mellitus 98

depletion 58

overload 56

requirements 107

resuscitation 107

Fragile X syndrome 17

Fructose intolerance 94

G Galactorrhea 31

Gastrointestinal injury 75

Genetic counseling 19

Genital anatomy, abnormal 26

Genital development, precocious, boy 16

Genitalia

ambiguous 50

internal, abnormal 32

Germinal failure, primary 22

Gigantism, cerebral 8,

Glucocorticoid deficiency 62

isolated 9

Glucocorticoids 7

Glucose 38

determination, rapid 94

intolerance 11

Glucose blood

fasting 96

random 96

Glucosuria 60

Glycosuria 102

Goiter 29, 76, 80, 82, 84

Goitrogens 84

Gonadotropin

deficiency 24,26

idiopathic 30 solated 22

organic 30

Graves disease 80

maternal, neonatal 82

Growth

abnormal 12

acceleration 9, 19, 52

hormone

deficiency $6,10,12,38$

childhood 6

classic 4

insensitivity 36,38

excess 8

insensitivity 38

syndrome 4

treatment 6

normal 36

rate 8

poor 44

rate, reduced 6

poor 6

slow 36

velocity, declining 48

Gynecomastia

false 20

idiopathic prepubertal 20

H Hair, axillary, pubic 19

Hashitoxicosis 80

Heart

disease, congenital 45

failure, congestive 62

Hearing 44

Height velocity 4

decreased 22, 23, 24

increased 8, 14, 15, 16, 18

normal $8,14,18,22,24$

Hemodialysis 60

Hepatocellular insult, acute 92

Hermaphrodite, true 40

Hirsutism 11, 30, 32, 98, 100

idiopathic 31

Histiocytosis X 55

Homocystinuria 8

21-Hydroxylase deficiency

mild 52

moderate 52

secondary 46,60

Hyperandrogenemia 32,34

Hyperandrogenism, idiopathic 32,34
Hypercalcemia 54, 68

neonatal 68

Hypercalcemic crisis 68

Hypercortisolism 11

Hyperglycemia 96, 100, 102

$\mathrm{mild} /$ moderate 100

severe 100

Hyperhidrosis 32

Hyperhydration 56

Hyperinsulinemia 11, 94

Hyperkalemia 64

Hyperlipidemia 11

Hypernatremia 60

essential 54

Hyperosmolar nonketotic state 100

Hyperphosphatemic rickets

autosomal-dominant 72

autosomal-recessive 72

calciuria 73

Hyperpigmentation 52

Hyperplasia, congenital adrenal 16, 64

Hyperprolactinemia 32

Hypertension 11, 46, 52

renovascular 46

Hyperthermia, malignant 64

Hyperthyroidism 8, 15, 68, 80, 84

neonatal 82

Hyperthyroxinemia 90, 92

autoantibody-associated 92

familial dysalbuminemic 92

Hypertrichosis 32

Hyperventilation 60

Hypocalcemia

neonatal 70

late 74

severe 74

Hypodipsia 60

Hypoglycemia 9, 10

infants and children 94

Hypogonadism 8

congenital 28

primary, other 20

Hypokalemia 54, 66

Hypomagnesemia 70, 74

Hyponatremia

hypervolemic 62

hypovolemic 62

Hypoparathyroidism, maternal 70

Hypopituitarism 39

yypophosphatemic rickets 72

Hypospadias 37, 38, 40 
Hypothyroidism 7, 10-12, 14, 16, 62, 84 acquired, other types 78

congenital 76, 90

hypothalamic pituitary 90

juvenile 78,90

primary 30

secondary, tertiary 76,78

Hypothyroxinemia 90

latrogenic reasons $20,56,60,62,66,68,70$ lleus 66

Immobilization 68

Incubator temperature 60

Infant

diabetic mother 74

feed, mistake in preparing 60

Infertility 44

Inflammatory bowel disease 27

Insulin

exogenous (factitious hypoglycemia) 94 requirements 102

Insulinoma 94

Intersex 26, 34, 40

Intestinal absorption defect 74

Intrauterine adhesions 28

lodine

deficiency 76,84

exposure 76

J Jansen's metaphyseal dysplasia 68 Jaundice 94

K Kallmann syndrome 29, 36 Ketoacidosis 100, 102, 106 Ketonemia 94

Ketones, smell of 106

Ketonuria 62, 94

acidemia 106

Ketotic hypoglycemia 94

Kidney malformation 45

L Laurence-Moon-Biedl syndrome 10 Laxative abuse 6

Leptin 10

Lethargy 106

Liddle syndrome 46

Lipodystrophy, total 8

Liquorice abuse 66

Liver

cirrhosis 56,62

disease 20,70

chronic 21

Lymphedema 45
M McCune-Albright syndrome 14

Macroglossia 94

Macro-orchidism, without virilization 16

Macrosomia, no maternal diabetes 94

Malabsorption 70, 72

Malnutrition 2

short gut syndrome 74

Mammoplasia, infantile 14

Marfan syndrome 8

Maternal deprivation 2

Menstrual disorder 11

Mental retardation 69

Micropenis 36, 38, 42, 94

Mineralocorticoid excess 46, 66

$5^{\prime}$-Monodeiodinase, generalized deficiency 92

Mumps orchitis 21

Muscular twitching 75

$\mathrm{N} \quad$ Nasogastric reflux/aspirate 66

Neonatal goiter 84

Neonatal rickets 72

Neonatal thyrotoxicosis 82

Nephrotic syndrome $56,62,70$

Neurofibromatosis 8

Neuroglycemia 95

Newborn screening tests 76

Nonpathogenetic stigmata 28

Nystagmus 94

Obesity $10,30,32,48,58,100$

infantile 10

mild truncal 11

simple 10

Oily skin 53

Oligomenorrhea 28,29

Omphalocele 9

Optic glioma 9

Orchidopexy 22

Orchitis 20

$$
\text { torsion } 22
$$

Organomegaly 9

Osmolality 62

Osteomalacia

tumor-induced 72

smotic diuresis 62

Otitis media 45

Ovaria 28, 30

Ovarian/adrenal tumor 34

Ovarian cyst/tumor 14

Ovarian failure, primary 24,28

Ovarian hyperandrogenism, functional 32

Ovaries

not polycystic 34 polycystic 34

Overweight 10

P Panhypopituitarism 36-38

Parents, tall, non-tall 8

Penile length, mean, stretched 17

Penis

large 19,52

size, increase 23

Peutz-Jeghers syndrome 21

Pheochromocytoma 46

renal 46

Phosphate deficiency 72

Pima Indian 100

Pituitary

disorder 6

multiple, hormonal deficiency 92

tumor 30

Pituitary/hypothalamic disease 6

Polycystic ovaries syndrome 10, 19, 32, 98, 100

atypical 34
classical 34

nonclassical 34

Polydipsia 102, 106

primary 62

Polyuria 54, 102, 106

Poor length gain 2

Prader-Willi syndrome 10

Prealbumin (transthyretin) hyperthyroxinemia 92

Precocious pubarche 18

Prediabetes, prediction of diabetes 98

Pregnancy 28

test positive 26

Prematurity 94

Prolactin 30

Prolactinoma 22

Pseudogynecomastia 21

Pseudohypoaldosteronism 64

Pseudohypocalcemia 70

Pseudohypoparathyroidism 10, 70

Psychological problems 102

Psychological stress 2, 29

Puberty 8, 102

abnormal 12

delayed 42

idiopathic central precocious 14

infantile central precocious 14

organic central precocious 14

peripheral (pseudo)precocious,

central (true) precocious 16

precocious, delayed 12

true, precocious 8

Pubic hair 53

absent, present 20

development 17 
R Rachitic bone changes 73

\section{Radiation}

exposure 88

no exposure 88

Renal disease 54

Renal failure 56, 62, 70

$$
\text { acute } 74
$$

Renal insufficiency, chronic 60

Renal tubular injury 75

Respiration deep-sighing, Kussmaul 106

Rheumatic disease 25

Rickets 70

calcitriol-resistant, vitamin D-dependent,

hypophosphatemic, hepatic 72

hypoparathyroidism 72

neonatal 72

X-linked hypophosphatemic, autosomal-recessive

hypophosphatemic with calciuria 72

Rokitansky syndrome 26

\section{S Salt-losing nephropathies 62}

Salt loss, renal 58

wasting, cerebral 54

Sarcoidosis 5

Scrotal size, texture, pigmentation 17, 19, 23

Sea water intoxication 60

Seborrhea 32

Seizure 94

Sex

characteristics, secondary 9

chromosome disorders 8

differentiation 39

Sexual precocity, central, peripheral 18

Shock 63, 106

volume depletion, acute 58

Short stature 4, 6, 23

female 44

idiopathic 4

Sleep apnea 11

Small for gestational age 74,94

Sodium overload 60

Steroidogenic block(s), 28

Steroids, exogenous 14

Sweatiest 62

Sweating 94
T $\quad \mathrm{T}_{4}$-binding protein abnormality 76

Tachycardia 80, 82, 94

Tall stature

familial 8

unexplained 9

Testes

small 10, 38

retractile, vanishing 42

two prepubertal 16

Testicular atrophy 36,38

Testicular development, delayed or absent 22

Testicular dysgenesis 38

Testicular failure, primary 22

Testicular volume 17

bilateral increase, unilateral increase 16

Testosterone-binding globulin

deficiency 76

increased concentration 92

Testotoxicosis 16

Thelarche, premature 14

Thyroglossal duct, abscess 85

Thyroid

adenoma 88

toxic 86

carcinoma 86,88

cyst, isolated 86

disease 82

ectopic 76

eutopic 76

hormone(s)

generalized resistance to 92

partial peripheral resistance 78

pituitary resistance 80

medullary carcinoma 86

nodule(s) 84

autonomous 80

children, adolescents 86

clinically euthyroid 86

multiple 87

storm 80

tender/painful swelling 84

tenderness 80

Thyroid-stimulating hormone receptor

activating mutation 80

Thyroid-stimulating hormone-secreting pituitary adenoma 80

Thyroiditis

autoimmune, with hypothyroidism 88

(juvenile) chronic lymphocytic 78, 80, 84, 87

increased incidence 45

subacute 80,84

suppurative 84
Thyrotoxicosis

biochemical 92

clinical 86,92

factitia 80

non-immune-mediated 83

Tingling 75

Toxicosis, hyperpyretic 58

Tremor 75

Tremulousness 94

Turner syndrome 24,44

Type 1 diabetes mellitus 100, 102

Type 2 diabetes mellitus 98,100

U Undernutrition 30

Undervirilized boy 40

Underweight 26

Urinary tract infection 50

severe 96

Uterine bleeding, dysfunctional 29

V Vagina

blind, isolated 26

congenital absence 27

Vaginal aplasia 26

Ventricular fibrillation 64

Vitamin D 25-hydroxylase deficiency 72

Virilization 18, 40

absent, partial or adult, but small testes 22

girl 40, 50

maternal 41

rapid 29

Vomiting 50, 58, 106

$$
\text { prolonged } 66
$$

W Water depletion 60

Weakness 106

Weight 46

gain

poor 2

loss 102,106

William's syndrome 68

Wolfram syndrome 54

\section{Index of Signs and Symptoms}




\section{Abbreviations}

$=$ Androstenedione

= Adrenocorticotropic hormon

= Alcohol dehydrogenase

= Androgen-insensitivity syndrome

= Apparent mineralocorticoid excess

= Aldosterone-producing adenoma

$=$ Antithyroid peroxidase

$=$ Bone age

$=$ Body Mass Index

$=$ Blood urea nitrogen

= Congenital adrenal hyperplasia

= Complete blood count

$=$ Constitutional delay of growth and

puberty

$=$ Cystic fibrosis

CG = Chorionic gonadotropin

$\mathrm{CHO}=$ Carbohydrate

$\mathrm{CIS} \quad=$ Carcinoma in situ

$\mathrm{CMO}=$ Corticosterone methyl oxidase

$\mathrm{CPP}=$ Central precocious puberty

$\mathrm{CRH}=$ Corticotropin-releasing hormone

CT $=$ Calcitonin

CVP = Central venous pressure

DCCT = Diabetes complications and

control trial

DDAVP $=1$-Deamino-d-arginine vasopressin

DHEAS = Dehydroepiandrosterone sulfate

DHT = Dihydrotestosterone

DI = Diabetes insipidus

DKA = Diabetic ketoacidosis

DOC = Deoxycorticosterone

DSH = Dexamethasone-suppressible

hyperaldosteronism

\section{E1 = Estrone}

ECF $\quad=$ Extracellular fluid

$\begin{array}{ll}\text { ERG } & \text { Extracellular fluid } \\ \text { E Electroretinography }\end{array}$

ESR = Erythrocyte sedimentation rate

$\mathrm{F}=$ Follicular

FAH = Functional adrenal hyperandrogenism

FNA = Fine-needle biopsy

$\mathrm{FOH} \quad=$ Functional ovarian hyperandrogenism

$=\alpha$-Fetoprotein

\begin{tabular}{|c|c|}
\hline $\begin{array}{l}\text { FPG } \\
\text { FPIR } \\
\text { FSH }\end{array}$ & $\begin{array}{l}=\text { Fasting plasma glucose } \\
=\text { First-phase insulin response } \\
=\text { Follicle-stimulating hormone }\end{array}$ \\
\hline $\begin{array}{l}\text { GAD } \\
\text { GH } \\
\text { GHBP } \\
\text { GHD } \\
\text { GHRH } \\
\text { GI } \\
\text { GnRH }\end{array}$ & $\begin{array}{l}=\text { Glutamic acid decarboxylase } \\
=\text { Growth hormone } \\
=\text { Growth hormone-binding protein } \\
\text { = Growth hormone deficiency } \\
\text { = Growth hormone-releasing hormone } \\
\text { = Gastrointestinal } \\
\text { = Gonadotropin-releasing hormone }\end{array}$ \\
\hline $\begin{array}{l}\text { hCG } \\
\text { HNF } \\
11 \beta-H S D \\
H t\end{array}$ & $\begin{array}{l}=\text { Human chorionic gonadotropin } \\
=\text { Hepatocyte nuclear factor } \\
=11 \beta \text {-Hydroxysteroid dehydrogenase } \\
=\text { Height }\end{array}$ \\
\hline $\begin{array}{l}\text { IAA } \\
\text { ICA } \\
\text { IGF-1 } \\
\text { IGFBP } \\
\text { IGT } \\
\text { IHA }\end{array}$ & $\begin{array}{l}=\text { Insulin autoantibodies } \\
=\text { Islet cell antibodies } \\
=\text { Insulin-like growth factor } \\
=\text { IGF-binding protein } \\
=\text { Impaired glucose tolerance } \\
=\text { Idiopathic hyperaldosteronism }\end{array}$ \\
\hline JDF & $=$ Juvenile Diabetes Foundation \\
\hline LH & $=$ Luteinizing hormone \\
\hline $\begin{array}{l}\text { MCT } \\
\text { MCV } \\
\text { MEN } \\
\text { MODY } \\
\text { MRI }\end{array}$ & $\begin{array}{l}=\text { Medullary carcinoma of thyroid } \\
=\text { Mean corpuscular volume } \\
=\text { Multiple endocrine neoplasia } \\
\text { = Maturity-onset diabetes of the young } \\
\text { = Magnetic resonance imaging }\end{array}$ \\
\hline $\begin{array}{l}\mathrm{nl} \\
\text { NOCAH } \\
\text { NSD1 }\end{array}$ & $\begin{array}{l}=\text { Normal } \\
=\text { Nonclassical adrenal hyperplasia } \\
=\text { Nuclear receptor set domain containing } \\
\text { protein } 1 \text { gene } \\
=\text { Nonsteroidal anti-inflammatory drugs }\end{array}$ \\
\hline $\begin{array}{l}\text { OGTT } \\
\text { OHD } \\
\text { OHP } \\
\text { OT }\end{array}$ & $\begin{array}{l}=\text { Oral glucose tolerance test } \\
=\text { Hydroxylase deficiency } \\
=\text { Hydroxyprogesterone } \\
=\text { Osmotic threshold }\end{array}$ \\
\hline $\begin{array}{l}\text { P } \\
\text { PCO } \\
\text { PCOS }\end{array}$ & $\begin{array}{l}=\text { Plasma } \\
=\text { Polycystic ovary } \\
=\text { Polycystic ovary syndrome }\end{array}$ \\
\hline
\end{tabular}

$\mathrm{PCR}=$ Polymerase chain reaction

$\mathrm{PE} \quad=$ Physical examination

PG = Prostaglandin

PPP = Peripheral precocious puberty

$=$ Plasma renin activity

PRL = Prolactin

PTH = Parathyroidhormone

PTHrp = PTH-related protein

PTU = Propylthiouracil

RDS = Respiratory distress syndrome

= Serum

SDS = Standard deviation score

SGA = Small for gestational age

SHBG = Sex-hormone-binding globulin

$\mathrm{SIADH}=$ Syndrome of inappropriate ADH secretion

$\mathrm{T} \quad=$ Testosterone

TBG = Thyroxin-binding globulin

TBPA = Thyroxin-binding prealbumin

= Type 1 diabetes mellitus

T2DM = Type 2 diabetes mellitus

TGAb = Thyroglobulin antibody

THE = Tetrahydrocortisone

THF = Tetrahydrocortisol

THS = Tetrahydro-11-deoxycortisol

TPOAb = Thyroid peroxidase antibody

TRAb = Thyrotropin receptor antibodies

TRH= Thyrotropin-releasing hormone

TSAb = Tubular resorption of phosphone

TSAb = Thyroid-stimulating antibodie

TSH = Thyroid-stimulating hormone

TSHR = Thyroid-stimulating hormone receptor

TSI = Thyroid-stimulating immunoglobulin

\section{U = Urinary}

$\mathrm{U} / \mathrm{L} \quad=$ Upper/lower

UFC = Urinary free cortisol

URTI = Upper respiratory tract infection

UTI = Urinary tract infection

$\mathrm{W} / \mathrm{U} \quad=$ Work-up

WBC = White blood cell count 